\title{
A Quantum to Classical Phase Transition in Noisy Quantum Computers
}

\author{
Dorit Aharonov*
}

October 9, 2018

\section{Abstract}

The fundamental problem of the transition from quantum to classical physics is usually explained by decoherence, and viewed as a gradual process. The study of entanglement, or quantum correlations, in noisy quantum computers implies that in some cases the transition from quantum to classical is actually a phase transition. We define the notion of entanglement length in $d$ dimensional noisy quantum computers, and show that a phase transition in entanglement occurs at a critical noise rate, where the entanglement length transforms from infinite to finite. Above the critical noise rate, macroscopic classical behavior is expected, whereas below the critical noise rate, subsystems which are macroscopically distant one from another can be entangled.

The macroscopic classical behavior in the supercritical phase is shown to hold not only for quantum computers, but for any quantum system composed of macroscopically many finite state particles, with local interactions and local decoherence, subjected to some additional conditions. This phenomenon provides a possible explanation to the emergence of classical behavior in such systems. A simple formula for an upper bound on the entanglement length of any such system in the super-critical phase is given, which can be tested experimentally.

\section{Introduction}

Quantum computation is a fascinating subject which manifests the peculiarities of quantum mechanics and uses them in order to achieve an advantage in terms of computational power over classical computers. Shor's algorithm 23] is the most astonishing known example for such an advantage in computational power: It enables one to factor an integer in polynomial time using a quantum computer, whereas the best classical algo-

\footnotetext{
*Computer Science division, UC Berkeley, E-mail: doria@cs.berkeley.edu
}

rithm for this task is sub-exponential. The advantage of quantum algorithms such as Shor's algorithm over classical algorithms suggests, though not proves, that the computational complexity class of quantum computation is not polynomially equivalent to that of classical, even randomized, Turing machines.

In real life, quantum computers will be subjected to noise. We will make here the assumption that the noise is probabilistic and local, meaning that each particle, each time step, suffers a certain faulty event with probability $\eta$, which is referred to as the noise rate. Quantum computing is now known to maintain its full computational power even in the presence of such local noise, as long as the noise rate is weaker than a certain threshold $\eta_{0}$ 3, 16, 17. On the other hand, it is known 2] that when the noise in the system is stronger than a much higher threshold, $\eta_{1}$, the quantum computation can be simulated efficiently by a classical Turing machine. Trying to put together the two results, we learn that there are two regimes of noise in the quantum computer, in which the computational power of the system is qualitatively different. For weak noise, the computational power is fully quantum, whereas for strong noise it becomes classical. This raises the following question: What is the physical difference between the two noise regimes, which reflects itself in the difference in computational power, and how does the transition between the two different physical behaviors occur?

It turns out that an answer to these questions can be given in terms of the behavior of entanglement, or quantum correlations in the system. Perhaps the best way to explain the notion of entanglement is by saying what entanglement is not: We say a state in the Hilbert space of a composite system $A \otimes B$ is non entangled, if two persons, Alice and Bob, each having access to one side of the system, could construct the overall state by applying local quantum operations on their side, and exchanging classical information, by, say, talking on the phone. Any state in the composite system $A \otimes B$ that cannot be constructed in this way is said to be entan- 
gled. Here we will be interested not only in whether or not states are entangled, but rather in the amount of entanglement in quantum states. Several possible definitions for the amount of entanglement has been suggested: The entanglement of formation[7], the asymptotic entanglement of formation [7] and the asymptotic entanglement of distillation [7, 22]. All these definitions are equally suitable for the purposes of this paper, and the results hold for any measure of entanglement which satisfies certain continuity requirements.

To study the behavior of entanglement in noisy quantum computers, we define the notion of entanglement length. Roughly speaking, the entanglement length is the rate of decay of the entanglement between two disjoint sets of particles, as a function of the distance between the two sets. This is the analogous quantity to correlation length in statistical physics, except that here we will be interested in correlations between two subsets of the system, rather than in two-point correlations. We study the behavior of the entanglement length in the noisy quantum computer as a function of the noise rate. We find that there exists a noise rate, $\eta_{1}$, which depends on the geometry of the system, such that the entanglement length is finite for $\eta_{1}<\eta \leq 1$. This means that the entanglement between two sets of particles decays exponentially with the distance between the two sets for this range of noise rates. On the other hand, the entanglement length is shown to be infinite in the range $0 \leq \eta<\eta_{0}$, where $\eta_{0}$ is the threshold for fault tolerance. This means that the entanglement between two sets of particles is independent of the distance between the two sets. These two facts show the existence of a phase transition in entanglement at a non-trivial noise rate $\eta_{0} \leq \eta_{c} \leq \eta_{1}$. The system in the sub-critical regime behaves quantumly even on the macroscopic scale- two sets of particles, within macroscopic distance, can share a lot of entanglement, so there is long range entanglement in the system. In the super-critical phase, where entanglement decays exponentially with the distance, the system behaves classically on the macroscopic scale, because two macroscopic subsets within macroscopic distance are practically non entangled.

The results here are by no means specific to quantum computers. In fact, we show that macroscopic classical behavior is expected above the critical noise rate for any macroscopic quantum system with local interactions, and local noise, where we make the additional assumption that there is time separation between two interactions in which one particle participates. This shows that a phase transition in entanglement is expected in any such quantum system which exhibits long range entanglement in the absence of noise. Moreover, our results can be verified experimentally in any such system, as long as the density matrices of subsystems can be measured accurately enough. The entanglement length can then be numerically computed (this is a difficult computational task, but possible for small subsystems) and compared to the finite entanglement length which is predicted by our analysis.

The emergence of classical macroscopic behavior in large quantum systems has been an intriguing area of research for the last several decades. Perhaps the most common and acceptable explanation so far is by decoherence, i.e. interactions with the environment which cause the system to lose its quantum features. See, for example, 24, 10] and references therein. This explanation, however, predicts a gradual transition from quantum to classical behavior. The most interesting implication of the results presented in this paper is that they suggest an alternative way to explain the transition from quantum macroscopic behavior to classical macroscopic behavior in certain physical systems, which is qualitatively different from the standard gradual explanation. The origin for the abrupt phase transition from quantum to classical predicted by our results is that in our model, we combine the decoherence process with the assumption that noise, as well as interactions, are local, where the behavior we are interested in is global.

The first part of the proof involves showing that the entanglement decays exponentially with the distance when $\eta$ is larger than a certain threshold. To do this, we use a method due to Aharonov and Ben-Or 22 to present the density matrix by mixtures of clustered states, and then we study the behavior of the sizes of the clusters, evolving in time, using a mapping of the problem to percolation. Known results from percolation theory 14 imply an exponentially decaying bound on the probability for distant sets of particles to be connected in the same cluster, and this implies exponentially small entanglement between the two sets. For the second part of the proof, i.e. in order to show that the entanglement length is infinite for weak noise, we use fault tolerant quantum computation, which enables one to create long range entangled states in the noisy quantum computer.

We start by defining the notion of entanglement, and entanglement length, and then proceed to prove the strong noise case and the weak noise case. We conclude with several open questions regarding possible implications to the transition from quantum to classical. 


\section{Entanglement}

The notion of entanglement is associated with a state of a quantum system composed of two systems, $A$ and $B$. The term entanglement refers to the quantum correlations between $A$ and $B$, in a state which lives in $A \otimes B$. Remarkably, two parts of a composite quantum system can exhibit very strong correlations which cannot be explained classically, unless one drops a very important axiom in physics, namely locality. The remarkable phenomena which can be exhibited due to entanglement between two quantum systems were first discovered by Einstein, Podolski and Rosen[11], more than 60 years ago, and manifested in Bell's inequality more than 30 years ago[ [4. However, the elusive phenomena of entanglement is still far from being understood.

In this paper we will be interested in the amount of entanglement in quantum systems; we therefore need a good measure of entanglement. One very important requirement on such a measure is that the entanglement in any state cannot increase by classical communication and local quantum operations on the $A$ and $B$ sides separately; this is the whole essence of the term entanglement. We will denote such a process involving local operations and classical communication by LOCC. A good measure of entanglement should also be additive.

A natural way to construct a measure of entanglement is to ask whether there is an elementary unit of entanglement, so that any state can be constructed with LOCC given sufficiently many such entanglement units. It turns out that there exists exactly such a unit: the Bell state, $\frac{1}{\sqrt{2}}(|0\rangle \otimes|0\rangle+|1\rangle \otimes|1\rangle)$. It was shown[6, 7 that any bipartite quantum state can be generated by Alice and Bob using only LOCC operations given that sufficiently many Bell states are a priori shared between $A$ and $B$. One can try to use the number of elementary units required to construct a state as a good measure of the entanglement in this state. It is reasonable to take the asymptotic limit of such a process, and to define the entanglement in a state as the following limit. Let $\phi$ be our quantum state, and let $k_{n}$ be the number of Bell states required to generate a state, $\phi_{n}$, and let $\phi_{n}$ approach the state $\phi^{\otimes n}$ as $n$ tends to infinity. Bennett et al[7] defined the infimum over all such processes, of $k_{n} / n$, as $n$ tends to infinity, as the asymptotic entanglement of formation of the state $\phi$. Let us denote this measure by $E_{f}^{\infty}$. This measure is clearly additive, and can also be shown not to increase by LOCC [7.

An equally natural definition would be the converse one, called the asymptotic entanglement of distillation, in which one is interested in generating as many Bell states as possible by applying LOCC on many copies of the original state $\phi$. The asymptotic limit of the ratio between the number of Bell states generated in this way, and $n$, was defined in $[7]$ to be the asymptotic entanglement of distillation. A more rigorous definition was given by Rains 22]. Let us denote this measure by $E_{d}^{\infty}$.

Fortunately, for pure states these two measures coincide, and have a very beautiful form. As was shown in [6], they are exactly the von-Neumann entropy of the reduced density matrix on one part of the system.

$$
E(A: B,|\phi\rangle\langle\phi|)=S\left(|\phi\rangle\left\langle\phi||_{A}\right) .\right.
$$

The entropy of entanglement thus possesses both additivity and monotonicity under LOCC, and also behaves nicely in many other ways.

The situation for mixed states, however, is much more interesting. It turns out that though the asymptotic distillable entanglement and the asymptotic entanglement of formation coincide on pure states, there are very interesting differences between them when mixed states are considered. Clearly, the asymptotic entanglement of distillation is not larger than the asymptotic entanglement of formation [7]. The question of whether there exist states in which $E_{f}^{\infty}$ is strictly larger than $E_{d}^{\infty}$ is still open. This irreversible process, in which not all of the entanglement which was inserted into the state can be distilled, is called bound entanglement 15 and is now being extensively studied.

The asymptotic entanglement of formation is believed to be equal to the following quantity, called the entanglement of formation $\left[\overline{[}\right.$, and denoted by $E_{f}$. $E_{f}(\rho)$ is the least expected entropy of entanglement of any ensemble of pure states realizing $\rho$, or more formally:

$E_{f}(A: B, \rho)=\min _{\sum_{i} w_{i}\left|\alpha_{i}\right\rangle\left\langle\alpha_{i}\right|=\rho} \sum_{i} w_{i} E\left(A: B,\left|\alpha_{i}\right\rangle\left\langle\alpha_{i}\right|\right)$.

The question of whether $E_{f}$ is equal or not to $E_{f}^{\infty}$ depends on whether $E_{f}$ is additive. It is believed that indeed it is the case that $E_{f}$ is additive, but this is not known.

Let us survey what is known about the above three entanglement measures, in terms of convexity and continuity. Entanglement of formation is trivially convex. Asymptotic entanglement of formation can also be shown to be convex, using the law of large numbers. Currently it is not known whether asymptotic distillable entanglement is convex or not. As for continuity, the situation is even less clear. It is known that the above three entanglement measures are continuous, in 
the sense that if a sequence of density matrices $\sigma_{n}$ converges to a density matrix $\rho$ in the trace metric, then the entanglement in $\sigma_{n}$ converges to the entanglement in $\rho$ :

$$
\lim _{n \longrightarrow \infty} \sigma_{n}=\rho \Longrightarrow \lim _{n \longrightarrow \infty} E\left(\sigma_{n}\right)=E(\rho) .
$$

However, we will be interested in how different can the entanglement of two very close density matrices be. Entanglement of formation was recently shown 20 to have very strong continuity properties, in the following sense: Given two density matrices of a bipartite Hilbert space of dimension $d \times d^{\prime}$, which are within $\epsilon$ distance one from another, in the trace metric, the entanglement of formation of the two matrices is at most $\epsilon$ times some linear function in $\log (d)$ and $\log \left(d^{\prime}\right)$, plus a term independent of $d$ and $d^{\prime}$ which goes to 0 as $\epsilon$ goes to 0 :

$$
\begin{aligned}
& \left|E_{f}(A: B \mid \rho)-E_{f}(A: B \mid \sigma)\right| \leq \\
& \left.9|\rho-\sigma| \log \left(\max \left\{d, d^{\prime}\right\}\right)-|\rho-\sigma| \log (|\rho-\sigma|)\right) .
\end{aligned}
$$

This strong continuity implies that when two density matrices of $n$ finite state particles are polynomially close one to another (in the number of particles), the entanglement of formation between them is also polynomially small. It is not yet known whether the asymptotic measures of entanglement possess these nice continuity properties, or not.

In this paper we work with the entanglement of formation, $E_{f}$, which is known to be both convex and strongly continuous. However, it should be stressed that the phenomena presented in this paper depend very weakly on the exact properties of the measure of entanglement which is being used. The results in this paper hold, with straight forward modifications, for any measure of entanglement $E$ which is continuous in a sufficiently strong sense, meaning that two density matrices which are $\epsilon$ apart have entanglements not different by more than $\epsilon$ times some polynomial in the number of particles.

\section{The Model of the Quantum System}

We are interested in quantum systems composed of $n$ two-state particles, embedded on a $d$-dimensional lattice. Such quantum particles are usually called qubits in the context of quantum computers. The Hilbert space of $n$ such particles is the tensor product of $n$ two dimensional complex vector space, $\mathcal{C}^{2}$, where the basis of $\mathcal{C}^{2}$ is standardly taken to be $|0\rangle$ and $|1\rangle$. The system is initialized with a certain state (usually a tensor product state, but not necessarily) and evolves in time via interactions between the particles. Time is discretized into time steps and all interactions are assumed to be instantaneous and occur at integer times. In this model, particles interact only with their nearest neighbors on the lattice. An important assumption is that one particle cannot interact with more than one other particle at a time. For simplicity, we will assume that the particles interact alternately with particles to each of their sides. For one dimension, i.e. an array of particles, this means that a particle interacts with a particle to its left and to its right alternately. The interaction graph can be easily viewed in a $d+1$ dimensional scheme, which for $d=1$ looks as follows:

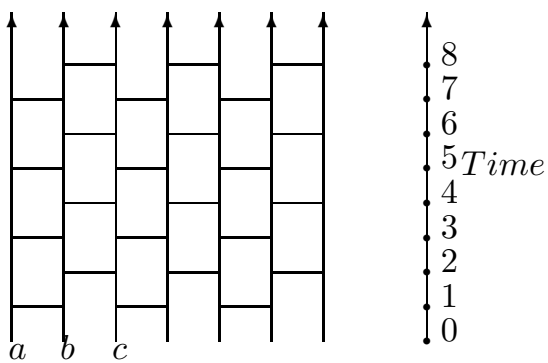

Figure 1: The vertical axis corresponds to time, and the horizontal axis corresponds to space. Horizontal edges connect two interacting particles. Particles interact alternately with particles to their left or to their right.

Two particles can interact via an arbitrary interaction, and we do not assume anything about the nature or strength of each interaction. After the interactions are turned off, and before the next step of interactions is turned on, we apply the noise step. The noise is assumed here to be local and stochastic, meaning that each particle with a certain probability $\eta$ undergoes an arbitrary fault process. As was shown in 13, 13 such $d$ dimensional noisy quantum circuits are capable of performing fault tolerant quantum computation, as long as the noise rate is smaller than a certain threshold. The threshold, however, is worse than the threshold without the nearest neighbor restriction, by one or two orders of magnitude, depending on the dimension.

We make here another assumption, and restrict the noise to be one of the following two processes. The first process, namely independent stochastic collapses, is a process in which at each time step, each particle is measured with independent probability $\eta$, in a fixed but arbitrary basis. Alternatively, we can use the depolarization model, in which at each time step, each 
particle, with independent probability $\eta$, is replaced by a particle in a completely mixed state. In the rest of the paper, we will assume that the noise model is independent stochastic collapses, but all results can be easily stated using stochastic depolarization.

It should be noted that the results of the paper hold when relaxing several of the assumptions we have made. The results apply to particles with any finite number of states, not necessarily qubits, and different particles need not have the same number of possible states. The exact form of the alternating interactions is not important, since any interaction graph of nearest neighbor interactions is a subgraph of the alternating graph, if time is scaled by a factor of $2 d$. The assumption of instantaneous interactions is also not essential, as long as the interactions last for less than a time step, so that there is a time interval in which decoherence takes place when the particle is not participating in any interaction. The results hold also in the case of noisy interactions, with some noise rate $\delta$. We will see that the proof of the upper bound on the entanglement length, for large $\eta$, holds regardless of the amount of noise in the interactions, $\delta$, because the proof only uses the noise occurring between interactions. The proof of the lower bound on the entanglement length, for small $\eta$, goes through as long as $\eta+\delta$ is smaller than the threshold for fault tolerance. Hence, it is straight forward to include in our model the above generalizations. For simplicity, however, we will work with the model defined above, of two state particles with noiseless instantaneous interactions.

\section{Entanglement Length}

For quantum systems which are embedded on a lattice, the notion of distance between sets of particles is well defined. In this case, one can define the entanglement length in the system. We would like to define an analogous quantity to the standard correlation length from statistical physics. In this case, one says that the correlation length in a physical system is $\xi$ if the correlation between the outcomes of a certain observable $O$ measured at sites $a$ and $b$, decays exponentially with the distance between them, $d(a, b)$, where the distance is scaled in the decay factor by $\xi$ :

$$
<O_{a} O_{b}>\propto e^{-\frac{d(a, b)}{\xi}}
$$

More precisely, $\xi$ is defined to be the following quantity:

$$
\xi^{-1}=\lim _{d(a, b) \longmapsto \infty}\left(\frac{-\log <O_{a} O_{b}>}{d(a, b)}\right) .
$$

In analogy with correlation length, we could have defined the entanglement length in the quantum system to be $\mu$ if the entanglement between two particles, $a$ and $b$, decays exponentially with the distance between them, $d(a, b)$, where the distance is scaled in the decay factor by $\mu$ :

$$
E(a: b) \propto e^{-\frac{d(a, b)}{\mu}} .
$$

However, there are a few problems with this definition, which will force us to modify it slightly. The first modification is necessary due to the fact that entanglement is a non-local quantity. It might well be that the system contains a lot of entanglement, but small subsets of the system are completely unentangled. For example, in fault tolerant quantum computers, the entanglement is bound to be shared by large sets of qubits, and in order to see entanglement it is necessary to probe large subsets of the system. We will therefore be interested not in two point correlations, but in entanglement between two sets $A$ and $B$ of arbitrary sizes.

Another problem is the following. In systems which are homogeneous in space and time, one can easily take the limit of the size of the system to infinity, and therefore the asymptotic behavior in equation (6) is well defined. However, we are interested in fault tolerant quantum computers, which are not homogeneous in space, nor in time. Roughly speaking, we will say that the entanglement length in the system is $\mu$ if the entanglement between any two sets $A$ and $B$ is bounded by a function which decays exponentially with the distance between the sets, where the decay factor is scaled by $\mu$. The fact that we are interested in a bound, and not in exact behavior of any pair of sets, allows for non homogeneity in space. To allow for non homogeneity in time, we will consider the average entanglement between $A$ and $B$ over the time from $t=0$ to $t=\infty$. This corresponds to the following behavior:

$$
<E(A: B)>_{t=0}^{\infty} \propto \operatorname{poly}(|A|,|B|) e^{-\frac{d(A, B)}{\mu}} .
$$

where $|A|$ is the number of particles in $A$ and similarly for $B$. We allow the additional polynomial factor due to the fact that for sets which are not too large compared to the distance between them, the exponential decay dominates the polynomial in the sizes of $A$ and $B$, and what we will see is merely an exponential decaying behavior. We claim that it is not reasonable to consider two sets of particles which are very large, and to study the behavior of the entanglement they share as a function of the distance between them, in the range where that distance is extremely small compared to the sizes of the sets. 
The characterization of $\mu$ by equation $(8)$ is very helpful to keep in mind. We can also make the definition of entanglement length more rigorous, by giving it a similar form to that of equation (6). This would be useful when one actually wants to calculate the entanglement length. In order to do that, we first need to make the notion of a quantum system more precise. In the non-homogeneous case, it is not clear what the notion of an infinite system means. We therefore define a quantum (infinite) system to be a sequence of quantum systems, $Q_{n}$, where $Q_{n}$ consists of $n$ particles. We think of $n$ as growing to $\infty$, but for a given $n Q_{n}$ is a finite system in space, which evolves in time from $t=0$ to $t=\infty$. Since each $Q_{n}$ is finite in space, in order to take a limit similar to that of equation (6), we need to consider a sequence of pairs of sets, $A$ and $B$ which belong to larger and larger systems. We thus add a subscript $n$ to the subsets $A_{n}$ and $B_{n}$, indicating that they belong to the quantum system $Q_{n}$. We would now like to translate the fact that we are interested in sets which are not too large compared with their distance to a precise restriction on the sequences of sets $\left\{A_{n}\right\},\left\{B_{n}\right\}$. The weakest condition which we can impose, to avoid pathologic cases, is that $\lim _{n \longmapsto \infty}\left|A_{n}\right| \cdot\left|B_{n}\right| / \exp \left(d\left(A_{n}, B_{n}\right)\right)=0$, meaning that the sizes of $A_{n}$ and $B_{n}$ are not growing exponentially or faster than exponentially with the distance between them. Finally, we want to take care of the fact that we are interested in the largest entanglement length which can be observed in the system. This corresponds to taking the infimum over all such sequences of $A_{n}$ and $B_{n}$. All this translates to the following definition:

Definition 1 The entanglement length $\mu$ of a quantum system $\left\{Q_{n}\right\}_{n=1}^{\infty}$ is defined by:

$\mu^{-1}=\inf _{\left\{A_{n}\right\}_{n=1}^{\infty},\left\{B_{n}\right\}_{n=1}^{\infty}} \liminf _{n \longmapsto \infty}\left(\frac{-\log <E\left(A_{n}: B_{n}\right)>_{t}}{d\left(A_{n}, B_{n}\right)}\right)$

where for all $n, A_{n}$ and $B_{n}$ are disjoint sets in $Q_{n}$, and the sequences $\left\{A_{n}\right\}_{n=1}^{\infty},\left\{B_{n}\right\}_{n=1}^{\infty}$ satisfy $\lim _{n \longmapsto \infty}\left|A_{n}\right|$. $\left|B_{n}\right| / \exp \left(d\left(A_{n}, B_{n}\right)\right)=0$.

Note that if one plugs into definition (1) the exponential behavior of equation (8), the contribution of the polynomial factor in equation (8) tends to zero due to the requirements on $A_{n}$ and $B_{n}$, and the correct $\mu$ pops out. Though definition (1) might seem complicated, calculating the above infimum turns out to be very simple in all our applications.

\section{Clustered Density Matrices}

We now proceed to study the entanglement length in $d$ dimensional noisy quantum circuits, in the strong noise regime. In this case, we will try to bound the entanglement in the system from above. Now a very useful observation is in place. After a particle was hit by the noise process, it is no longer entangled with the rest of the system. In other words, in both noise processes which we consider, the density matrix after applying the noise process can be written in the form

$$
\mathcal{E} \rho=(1-\eta) \rho+\eta \sum_{i} p_{i} \rho_{i}^{Q} \otimes \rho_{i}^{q}
$$

where the index $q$ refers to the noisy particle, and $Q$ refers to the rest of the system. For example, for the stochastic noise process, in which the last qubit is measured in the basis $\{|0\rangle,|1\rangle\}$, the resulting density matrix would be of the form:

$$
\mathcal{E} \rho=(1-\eta) \rho+\eta \sum_{i=0}^{1} \operatorname{Pr}(i) \rho_{i}^{Q} \otimes|i\rangle\langle i|
$$

where $\rho_{i}^{Q}$ is the density matrix of all but the last qubit, under the condition that the last qubit is measured to be in the state $|i\rangle$. We use this observation as follows. We will aim to present the density matrix in such a way that lack of entanglement translates to tensor product structure. In other words, we will present a density matrix as a mixture of tensor product states, as follows:

$$
\begin{aligned}
\rho(t) & =\sum_{i} w_{i} \rho_{i}(t), \\
\rho_{i}(t) & =\rho_{i}^{1}(t) \otimes \cdots \rho_{i}^{m_{i}}(t)
\end{aligned}
$$

where $\rho_{i}^{j}(t)$ is a density matrix which describes a set of particles $A_{i}^{j}$, and for each $i$ the sets $A_{i}^{j}$ are a partition of the system. These sets of supposedly entangled particles are called clusters. It should be understood here that given a density matrix, there is no single way to present it as a mixture of clustered states. However, we will define the representation according to the dynamics of the process which generated $\rho$, so that our representation will be well defined.

Our goal would be to find a way to represent the matrix as a mixture of clustered states with as small clusters as possible. The intuition is that we want to give an upper bound on the amount of entanglement in the system. When all the clusters are of size one, there is no entanglement in the system. We will see later, that this can be generalized to say that small localized clusters imply no entanglement between distant sets. We will thus try to keep the clusters as small as 
possible. The way we do this is as follows. In a quantum computer, the initial state is a basic state, which is a pure state in which all qubits are in tensor product with one another:

$$
\rho=\rho(1) \otimes \rho(2) \otimes \cdots \otimes \rho(n) .
$$

Thus, for $t=0$, all clusters are of size 1. Given any clustered states description of the density matrix at time $t$, we can obtain a clustered state description for the matrix at time $t+1$ as follows. From each participant in the mixture, $\rho_{i}(t)$, we obtain $\rho_{i}(t+1)$ which will be a mixture of clustered states. $\rho(t+1)$ will then be a mixture of all $\rho_{i}(t+1)$. To obtain $\rho_{i}(t+1)$ from $\rho_{i}(t)$, we first apply the interaction step, and then apply the noise step.

To apply the interaction step of time $t$, we apply for each interaction at that time step the unitary transformation corresponding to the interaction, on the appropriate pair of particles. If the two particles are from one cluster, then we simply apply the appropriate unitary matrix, corresponding to the interaction, on the density matrix describing this cluster, and there is no need to change the clusters. However, if the two particles are from two different clusters, we can no longer keep the two clusters in tensor product, because in general they will be entangled. Therefore, we first join the two clusters together, by taking the tensor product of the density matrices describing the two clusters, and then apply the appropriate unitary matrix on the new big cluster. The resulting state after all interactions of time step $t$ were applied is therefore, in general, a clustered state with larger clusters than the state $\rho_{i}(t)$.

We then apply the noise step on the resulting clustered state. Recall that a measurement detaches a particle from its cluster, and thus after a measurement the particle is a cluster of its own. To apply the noise process, we transform the state to a mixture of states, which are the results of all possible combinations of which particles where measured, with the appropriate probabilities. Clusters in the state can only shrink due to this process.

We would now like to understand the typical size of clusters in this representation of the density matrix. Before we do that in a more formal way, let us gain some intuition. If the system were noise free, very soon all the clusters would become one giant cluster of $n$ particles. What makes the situation more interesting are the stochastic collapses, which separate a measured particle from its cluster, thereby decreasing the size of the cluster by one, and creating another cluster of size one. One can view the noisy quantum evolution in time as a struggle between two forces: The interactions, which tend to join clusters and entangle the different parts of the system, and the stochastic collapses, which tend to detach particles from their clusters, thereby destroying this entanglement constantly. A crucial point here is that the two competing forces are matched in power, since they both operate on a linear number of particles $\theta(n)$ each time step. We thus expect a critical error rate, $\eta_{c}$, at which the two forces are equal, and at which some transition between the dominance of the entangling interaction process transforms to the dominance of the disentangling noise process. We now go on to see this phenomenon more rigorously, using a map to a percolation process.

\section{The Percolation Process}

It turns out that the dynamics of the clusters in the above description are intimately connected with a percolation process on the quantum circuit itself. The percolation process on the graph is defined as follows: For each time step, each vertical edge, along the $i^{\prime}$ th wire, between time $t$ and $t+1$, is erased with independent probability $\eta$. In the cluster picture, this corresponds to the collapse of the $i$ 'th particle between time steps $t$ and $t+1$, which disentangles the particle's past and future. Thus an event in the probability space of the noise process, i.e. a specific choice of which particles collapsed during the process, is mapped to an event in the percolation process, in which the corresponding vertical edges are erased. Since events in the stochastic noise process correspond to members in the mixed density matrix, we have a map between clustered states arising from our cluster dynamics, and realizations of the percolation process. This map preserves the probability measure.

We now claim that clusters in the clustered state correspond to connected components in the percolation process:

Lemma 1 Correspondence Lemma: Two particles $a$ and $b$ are in the same cluster at time $t$, in one realization of the noise process in the cluster model, iff $(a, t)$ and $(b, t)$ are connected in the corresponding realization of the percolation model.

Proof: To prove this combinatorial lemma we use induction on $t$. For the base of the induction, $t=0$, the correspondence is true by definition. Let us now assume that the lemma is correct for $t$, and prove for $t+1$. To apply the induction step, the following observation comes in handy. Each path that connects $(a, t+1)$ and $(b, t+1)$ in the percolation process, is actually a concatenation of alternating paths, occurring either after 
time $t$ or at times up to $t$. We denote the points at which the different concatenated paths connect one to another by $\left(x_{1}, t_{1}\right), \ldots,\left(x_{2 k}, t_{2 k}\right)$. It is easy to see that there is always an even number of such points, and that $t_{1}=t_{2}=\ldots=t_{2 k}=t$. Let us call the particles $x_{1}, \ldots, x_{2 k}$ the connection particles. We shall also denote $a=x_{0}, b=x_{2 k+1}$. A schematic example for the one dimensional quantum circuit case is shown in figure 2 .

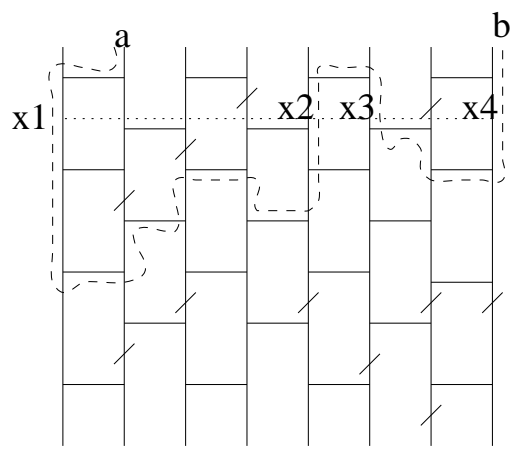

Figure 2: A path connecting the two particles, $a$ and $b$, at time $t+1$ can be represented as a concatenation of paths which are restricted alternately to the time intervals $[0, t]$ and $[t, t+1]$. The path $(a, t+1) \longmapsto(b, t+$ $1)$ in the figure is a concatenation of the paths $(a, t+$ $1) \longmapsto\left(x_{1}, t\right) \longmapsto\left(x_{2}, t\right) \longmapsto\left(x_{3}, t\right) \longmapsto\left(x_{4}, t\right) \longmapsto$ $(b, t+1)$.

Let us now prove the first direction: Let $a$ and $b$ be two particles connected at time $t+1$ in the percolation model. We want to show that $a$ and $b$ are in the same cluster at time $t+1$. To see this, we show that all particles $a=x_{0}, x_{1}, . ., x_{2 k}, x_{2 k+1}=b$ are in the same cluster at time $t+1$. For the pairs of particles $x_{2 i+1}$ and $x_{2 i+2}$, i.e. pairs in which the first particle has odd index, this is true since they are connected by a path confined to time steps $t$ or earlier, so by the induction assumption, they are in the same cluster at time $t$. Moreover, none of the connection particles collapsed between time steps $t$ and $t+1$, due to the fact that they connect between a path before time $t$ and a path after time $t$ (or vice-versa). Therefore $x_{2 i+1}$ and $x_{2 i+2}$ are in the same cluster also at time $t+1$. (In the schematic example, this shows that particles $x_{1}$ and $x_{2}$ are in the same cluster at time $t+1$, and similarly $x_{3}$ and $x_{4}$.) Now by definition of the connection particle, there is a path after time $t$ connecting $x_{2 i}$ and $x_{2 i+1}$, which means that $x_{2 i}$ and $x_{2 i+1}$ interact at time $t+1$. At the edges of the chain, i.e. for $i=0$ or $i=k$, it might be that $x_{2 i}$ and $x_{2 i+1}$ are the same particle, and therefore they are trivially connected. (In the example, this corresponds to the interaction at time $t+1$ between $a$ and $x_{1}$, and between $x_{2}$ and $x_{3}$, and to the fact that $b=x_{4}$. ) By the definition of the clusters' evolution in time, the fact that particles interact imply that their clusters are joined, and therefore $x_{2 i}$ and $x_{2 i+1}$ are in the same cluster at time $t+1$. Combining this with the fact that $x_{2 i+1}$ and $x_{2 i+2}$ are connected at $t+1$, this implies that all the particles $a=x_{0}, x_{1}, . ., x_{2 k}, x_{2 k+1}=$ $b$ are in the same cluster at time $t+1$, which completes one direction of the induction step.

Let us now prove the other direction of the induction step. We want to prove that there is a path connecting $(a, t+1)$ and $(b, t+1)$ in the percolation process, assuming that $a$ and $b$ are in the same cluster at time $t+1$. This cluster of time step $t+1$, which contains $a$ and $b$, was generated by joining together several smaller clusters, which existed after the noise step of time step $t$. It is easy to see that there is a subset of those clusters, $C_{-1}, C_{0}, \ldots, C_{k-1}, C_{k}$, such that $a \in C_{-1}$ and $b \in C_{k}$, and such that each two subsequent clusters, $C_{i}$ and $C_{i+1}$, were connected at time $t+1$ by a unitary gate. Note that each cluster $C_{i}$, except maybe $C_{-1}$ and $C_{k}$, consists of at least two particles: one particle, denoted by $x_{2 i+1}$, participates in an interaction with a particle from the preceding cluster in the chain, $C_{i-1}$, and the other particle, $x_{2 i+2}$, participated in an interaction with a particle from the next cluster in the chain, $C_{i+1}$. To construct a path from $a$ to $b$ at time $t+1$, we first note that by the induction assumption, there is a path connecting the two particles in the same cluster, $x_{2 i+1}$ and $x_{2 i+2}$, at time $t$. Moreover, these particles did not collapse between time step $t$ and $t+1$, because if they did collapse, they would have belonged to a cluster consisting of one particle exactly. Hence there is a path connecting them at time $t+1$. A path between the second particle of $C_{i}$ and the first particle of $C_{i+1}$ exists because by definition they interact at time $t+1$. Paths from $a$ to the first particle $x_{1}$, and from the last particle $x_{2 k}$ to $b$, exist either because they interact at time $t$, or they are simply the same particle. This enables us to construct a concatenated path from $a$ to $b$ at time $t+1$.

We can now investigate the sizes of the connected components in the percolation model and then translate our findings to the cluster model. Such percolation processes are known to exhibit a phase transition at a critical point, below which there is a connected component of linear size, but above which the typical connected components is of logarithmic size. Moreover, the connected components in the super-critical phase are localized, in the sense that the probability for two particles to be in the same connected component decays exponentially with the distance between these particles.

Let us first release the restriction to a percolation 
on a square of size $n \times T$ and consider the infinite lattice. We show the existence of a phase transition in connectivity for percolation on the infinite lattice, and from this we will be able to get information about the finite case. One more simplification which is useful is to notice that by contracting each edge which corresponds to an interaction to one point, we do not change the connectivity properties of the process, and the resulting percolation process is the usual model of percolation. For example, in the two dimensional lattice associated with the one dimensional quantum circuit, the interaction edges are exactly the horizontal edges in figure 1 , and so after contracting each of these edges to one point, the resulting percolation process is the standard percolation on the square lattice, which is rotated by 45 degrees. The contraction therefore transforms the problem to standard bond percolation on translational invariant lattices, which is pretty well understood.

In bond percolation on translational invariant lattices, one usually uses $p$ as the probability for one edge to be present, so in our case $p=1-\eta$. One can define the critical $p, p_{c}$, to be the smallest probability in which the point 0 belongs to an infinite connected component with positive probability. In translational invariant lattices, this is also equal to the smallest $p$ in which the expected size of $0^{\prime}$ s connected component is infinite 19, 1, 8

$$
\begin{aligned}
p_{c} & =\inf \left\{p \mid \operatorname{Pr}_{p}(|H(0)|=\infty)>0\right\}= \\
& =\inf \left\{p \mid E_{p}(|H(0)|)=\infty\right\} .
\end{aligned}
$$

where $H(0)$ is the connected component of 0 .

A theorem by Hammersley 8,14 , asserts that for translational invariant lattices, for $p<p_{c}$, the probability $\tau(x, y: p)$ for $x$ to be connected to $y$, decays exponentially with the distance:

$$
\tau(x, y: p) \leq \exp \left(-\frac{d(x, y)}{\xi(p)}\right)
$$

with $\xi(p)<\infty$. Above $p_{c}$, the probability for 0 to be connected to infinity is larger than 0 by definition of $p_{c}$, so $\xi(p)=\infty$ for $p>p_{c}$.

Let us denote by $p_{c}(d+1)$ the critical $p_{c}$ for bond percolation on the lattice corresponding to a quantum circuit of dimension $d$. For one dimensional quantum systems, it is easy to calculate the critical probability $p_{c}(1+1)$. After contracting the interaction edges, we simply get percolation on $Z^{2}$, for which it is known 14 that the critical probability is a half. Hence:

$$
p_{c}(1+1)=\frac{1}{2} \text {. }
$$

For higher dimensions, we can bound $p_{c}(d+1)$ away from 0 and 1 , but the bounds are not tight:
Lemma $2 \frac{1}{3} \leq p_{c}(d+1) \leq \frac{1}{2^{1 / d}}$.

Proof: The upper bound comes from the fact that the projection of the $d+1$ percolation with parameter $p$ on a $1+1$ percolation gives percolation in $1+1$ with parameter $p^{d}$. This is true since after a particle interacts with a particle along one axis, it waits $d$ time steps before it interacts again with a neighbor along the same axis. This gives the upper bound, since if the original process had exponentially decaying correlations, it cannot be that in the projected process we are above the phase transition where 0 is connected with constant probability to infinity. The lower bound is derived from a standard argument which reduces the problem to a branching process. A branching process is a process which starts with one node, and each nodes gives birth to $k$ nodes with some probability distribution $p(k)$, independent of the other nodes. It is a standard result (See [12], for example) that when the expected number of descendents for each node is less than 1, the dynasty dies in finite time with probability 1 . To construct the corresponding branching process, observe that the degree of the interaction graph, after contracting the horizontal edges, is exactly 4 , regardless of $d$. Starting with the point 0 , we regard each of its neighbors to which it is connected in the percolation as an ancestor of a dynasty. The descendents of each such node are all its neighbors, except for 0 , in the percolation process; Each descendent has its own descendents, and so on. When we encounter a node which is already in the dynasty, we do not count it again; this way we have a tree. Clearly, if the branching process is finite, then the connected component of 0 in the percolation process is definitely finite. However, each neighbor of 0 is the ancestor of a dynasty in which the expected number of descendents of each node is exactly the number of its neighbors which are not yet in the dynasty, times $p$. Since the degree of the graph is 4 , and one of the neighbors is the node's ancestor, the expected number of descendents is at most $3 p$, which is less than 1 for $p$ less than $\frac{1}{3}$. This gives the desired result.

The analysis of the percolation process has taught us that when $p$ is smaller than the critical point for percolation, $p_{c}$, the connected components in the system are small. Going back to the density matrices, using the correspondence lemma, this implies that for $\eta<1-p_{c}$, the clusters are small and localized. This will be used in the next section to prove an upper on the entanglement between distant sets in this noise regime. 


\section{$7 \quad$ Finite Entanglement Length}

From the correspondence to percolation, we have that for $\eta<1-p_{c}$, the density matrix of the quantum system can be approximated by a mixture of clustered states with localized clusters of logarithmic size. Thus, distant subsets of particles are with high probability contained in non-intersecting clusters, i.e. most of the weight of the density matrix is concentrated on states in which there is no entanglement between the two subsets. The weight of the states in which there is entanglement between the two sets decays exponentially with the distance between the sets. By continuity of entanglement, this implies that the entanglement between the two sets decays exponentially with the distance.

We can now show, that the entanglement between any two sets of particles decays exponentially with the distance between them, when the noise rate $\eta$ is such that $1-\eta$ is sub-critical in the percolation process. The rate of the decay is the entanglement length of the system. The entanglement between the two sets becomes negligible already when the distance is of the order of $\log (n)$ particles. This translates to the following theorem:

Theorem 1 Consider a d dimensional quantum circuit with nearest neighbor interactions, subjected to local noise of the type of stochastic depolarization or stochastic collapses, with noise rate $\eta$. If the circuit is initialized with an unentangled state, i.e. a tensor product state, and if $\eta>1-p_{c}(d+1)$, then the entanglement of formation between any two sets of qubits $A$ and $B$ at any time $t \geq 0$ decays exponentially with the distance between the two sets:

$$
E_{f}(A: B) \leq \min \{|A|,|B|\}|A| \cdot|B| e^{-\frac{d(A, B)}{\xi(1-\eta)}} .
$$

For a general initial state, a similar formula is true except for a correction term which decays exponentially with time:

$$
\begin{aligned}
E_{f}(A: B, \rho(t)) \leq & \min \{|A|,|B|\}\left(|A| \cdot|B| e^{-\frac{d(A, B)}{\xi(1-\eta)}}+\right. \\
& \left.+n \min \{|A|,|B|\} e^{-\frac{t}{\xi(1-\eta)}}\right) .
\end{aligned}
$$

Proof: Let us start with the simple case, in which the initial state is a complete tensor product, i.e. all clusters are of size 1. By equation 14, the probability for two particles $A$ and $B$ to be connected decays exponentially in the distance between them. The correspondence lemma (1) implies that the probability for two particles to be in the same cluster at time $t$ is equal to the probability they are connected in the percolation model at time $t$. i.e. the probability for two particles from $A$ and $B$ to be in the same cluster is bounded above by $\exp (-d(A, B) / \xi(1-\eta))$. Thus, the probability for any pair of particles from $A$ and $B$ to be in the same cluster is bounded above by $|A| \cdot|B| \exp (-d(A, B) / \xi(1-\eta))$. The density matrix can thus be written as a mixture of one density matrix with weight smaller than $|A| \cdot|B| \exp (-d(A, B) / \xi(1-\eta))$, and another density matrix which is a mixture of density matrices, where in all these matrices all the particles in $A$ are in different clusters than all the particles in $B$. The reduced density matrix to $A, B$ of the second matrix is thus separable, and contains no entanglement between $A$ and $B$. By convexity of entanglement of formation, the entanglement in the entire density matrix is bounded above by the entanglement in the first density matrix, times the weight of this matrix. The entanglement of the first matrix is at most the number of qubits in the system, and this gives the desired result. (For measures of entanglement which are not convex, but strongly continuous, one should replace the term $\min \{|A|,|B|\}$ by the appropriate polynomial from the continuity bound.)

We now proceed to the general initial state. We will give an upper bound for the case in which the initial state is one big cluster, and any other case is trivially implied by it. To do this, we have to understand where we have used the fact that the initial state is not entangled. This was used for the base of the induction in the correspondence lemma, where the fact that all clusters are of one qubit corresponds to the fact that in the percolation graph, the initial connected components at time 0 are all of size 1 . To adapt the situation to the case in which all particles are in one big cluster at time 0 , we add a horizontal line of length $n$ connecting all particles to one big connected component at time $t=0$. The correspondence lemma then goes through. However, equation 14 no longer holds. To correct it, we add to it a term which corresponds to the probability for $A$ to be connected to $B$ by a path that goes through time $t=0$, i.e. through the additional new line we have added to the graph. For such a path to exist, both $A$ and $B$ need to be connected to time 0 . The probability for any one of the qubits in $A$ to be connected to any one of the $n$ qubits at time 0 is at most $n|A|$ times the probability for one qubit at time $t$ to be connected to one qubit at time 0 , which is at most $\exp \left(-\frac{t}{\xi(1-\eta)}\right)$ by equation 14 . The same argument applies for the connection from $B$ to time 0 , and this gives the desired result.

This shows that the system cannot create entanglement between far sets of particles: Roughly speaking, the typical range of entanglement is microscopic. This 
is true for any initial condition, where the relaxation time to the typical unentangled state is of the order of $\log (n)$ steps.

This result implies an upper bound on the entanglement length in the quantum system above the critical noise rate, and in particular shows that it is finite. This is done by simply taking the limit in the definition of entanglement length (11), which gives:

Corollary 1 The entanglement length $\mu(\eta)$ of a $d$ dimensional quantum circuit with nearest neighbor interactions, subjected to local noise of the type of stochastic depolarization or stochastic collapses, with noise rate $\eta$, satisfies:

$$
\mu(\eta) \leq \xi(1-\eta)
$$

and in particular $\mu(\eta)$ is finite for $\eta>1-p_{c}(d+1)$.

This gives a bound on the entanglement length, in terms of the correlation length in classical bond percolation. The correlation length of a given lattice can be easily estimated by computer experiments, and analytical bounds are given in [8, 14].

\section{Infinite Entanglement Length}

We now want to concentrate on the other noise regime, and show that below the critical noise the entanglement length is infinite. One might naively think that this can be deduced from the fact that the density matrix is a mixture of clustered states with linear sized clusters. However, there is a difficulty in pursuing the connection between clusters and entanglement for this purpose, because of the following reason. The density matrix is actually a mixture of many clustered states. The mixture of two clustered states, with very large clusters, can be a density matrix in which the clusters are of size one. One example of such a case is a mixture of the two states, $\frac{1}{\sqrt{2}}\left(\left|0^{n}\right\rangle+\left|1^{n}\right\rangle\right)$ and $\frac{1}{\sqrt{2}}\left(\left|0^{n}\right\rangle-\left|1^{n}\right\rangle\right)$, the mixture of which is a non entangled state. Thus, the sizes of the clusters can be used for upper-bounds on entanglement, but it is not clear how to use them in order to show a lower bound on the entanglement in the system.

We therefore need to use different techniques for lower bounds on entanglement. We will use techniques from quantum computation. A quantum computer embedded on a lattice is a special case of the quantum systems we are discussing. The particles are quantum bits, and the interactions are fixed according to the algorithm. Therefore, corollary in shows that the entanglement length is finite above the critical noise rate also in fault tolerant quantum computers. For fault tolerant quantum computers we can also analyze the other side of the noise scale, and show that the entanglement length in the system is infinite if the noise rate is below a certain threshold. We will use the threshold result 3, 16, 17] for fault tolerant quantum computation, which shows that quantum computation can be made robust to noise, using quantum error correcting codes, as long as the noise is smaller than a certain threshold. In fact, here we need the slightly stronger version of the threshold result [3, 13], which asserts that this can be done even when the quantum system is embedded on a $d$ dimensional lattice. The threshold is then $\eta_{0}(d)$, which for $d=1$ is estimated to be $10^{-7}$ [3]. In the fault tolerant range, two distant sets of qubits can be entangled, and remain entangled for a long time, with the amount of entanglement independent of the distance.

We now give an example of a quantum computer which exhibits entanglement among far parts of the system when $\eta<\eta_{0}(d)$, but the entanglement length is finite for noise $\eta>1-p_{c}(d+1)$. The idea is that a fault tolerant computer can simulate any quantum state, including states which contain entanglement between sets of qubits which are far apart. Hence, we will construct a quantum algorithm in which there is entanglement between two far parts of the system, and make it fault tolerant. This can be done in many ways, but here is a simple example, for $d=1$. Divide the set of qubits to three sets, $A, B, C$. We will create entanglement among $A$ and $B$, while leaving the qubits in the middle, $C$, in a basic state. This will be done by constructing the state:

$$
\frac{1}{\sqrt{2}}\left(\left|0^{m}\right\rangle_{A} \otimes\left|0^{n}\right\rangle_{C} \otimes\left|0^{q}\right\rangle_{B}+\left|1^{m}\right\rangle_{A} \otimes\left|0^{n}\right\rangle_{C} \otimes\left|1^{q}\right\rangle_{B}\right)
$$

on a fault tolerant quantum computer, and keeping this state for a long time, by applying error corrections. This state indeed contains entanglement between the two registers $A$ and $B$, which are $n$ sites apart. The algorithm which constructs such a state is very simple, and uses only two basic quantum gates: The Hadamard gate, which is a one qubit gate applying the following unitary transformation

$$
\begin{aligned}
|0\rangle & \longmapsto \frac{1}{\sqrt{2}}(|0\rangle+|1\rangle) \\
|1\rangle & \longmapsto \frac{1}{\sqrt{2}}(|0\rangle-|1\rangle),
\end{aligned}
$$

and the controlled NOT gate which is a two qubit gate applying the following unitary transformation (said to be applied from the first qubit to the second one):

$$
|a\rangle \otimes|b\rangle \longmapsto|a\rangle \otimes|a \oplus b\rangle,
$$


where $\oplus$ means addition mod 2 . Using these gates, it is easy to create the state

$$
\frac{1}{\sqrt{2}}\left(\left|0^{m+q}\right\rangle+\left|1^{m+q}\right\rangle\right)
$$

on the first $m+q$ qubits, by applying a Hadamard gate on the first qubit and then controlled NOT gates from the first qubit to the second, from the second to the third, and so on. Then, we want to swap the $m+1, \ldots, m+q$ qubits to register $C$. To do this, we first swap the last qubit in $B$ with qubits to its right until it gets to the last site in $C$; In the same way we bring the one before last qubit in $B$ to the one before last site in $C$, and so on until all qubits in $B$ are in the right most sites of $C$, which achieves the desired state with only nearest neighbor interactions.

This algorithm by itself is not fault tolerant, and in the presence of any amount of noise, i.e. $\eta>0$, the entanglement in the system will be lost immediately. However, we can make this algorithm fault tolerant by the methods in 13, 16, 17, as long as $\eta$ is smaller than $\eta(d)$, the threshold for fault tolerance for $d$-dimensional quantum computers 3 , 13]. These results are too complicated to explain here in details. In a nutshell, fault tolerance is achieved by encoding the qubits using quantum error correcting codes, and computing the algorithm on the encoded states, while applying quantum error correction on the state frequently. Each qubit is replaced by polylog $(n)$ qubits, encoding its state. The state $|0\rangle$ is encoded by the state $\left|S_{0}\right\rangle$ of polylog $(n)$ qubits, and similarly $|1\rangle$ is encoded by the state $\left|S_{1}\right\rangle$. Let us denote by $A^{\prime}, B^{\prime}$ and $C^{\prime}$ the qubits encoding the original sets of qubits $A, B$, and $C$, respectively. If no fault occurs, at the end of the algorithm the state of the system will be in the state (16) encoded by the quantum error correcting code:

$$
\left|S_{0}^{m}\right\rangle_{A^{\prime}} \otimes\left|S_{0}^{n}\right\rangle_{C^{\prime}} \otimes\left|S_{0}^{q}\right\rangle_{B^{\prime}}+\left|S_{1}^{m}\right\rangle_{A^{\prime}} \otimes\left|S_{0}^{n}\right\rangle_{C^{\prime}} \otimes\left|S_{1}^{q}\right\rangle_{B^{\prime}}
$$

normalized by a factor of $\frac{1}{\sqrt{2}}$. The entanglement in this state will remain there for ever if errors do not occur. However, errors do occur. By fault tolerance, this means that at the end of the computation the density matrix is polynomially close to a density matrix $\rho$ which can be corrected to the correct state (20) by noiseless quantum error corrections. Due to continuity of entanglement, it suffices to argue that such $\rho$ contains a constant amount of entanglement. But this is true since we know that $\rho$ can be corrected to the state (20) by local operations not involving interactions between $A^{\prime}$ and $B^{\prime}$. Since entanglement cannot increase by local operations, the entanglement between $A^{\prime}$ and $B^{\prime}$ in $\rho$ is at least as that in the state encoding (20), i.e. one entanglement unit. The distance between the ac- tual density matrix and a correctable density matrix $\rho$ is, by [3], at most the number of time steps $t$ divided by a polynomial in $n$. This distance is smaller than some constant $\epsilon$ as long as the number of time steps is polynomial in the size of the system $n$. Thus, by strong continuity the entanglement between $A^{\prime}$ and $B^{\prime}$ will remain bounded from below by a constant for polynomially many time steps. After polynomially many steps, we can replace all qubits by qubits in the state $|0\rangle$, and run the whole algorithm again. The average entanglement over time from 0 to $\infty$ is very close to one, since the time it takes to construct the state is much smaller than the polynomial time for which the entanglement remains in the system. This proves the existence of a non trivial sub-critical side of the phase transition:

Theorem 2 The entanglement length in the d dimensional fault tolerant quantum computer defined above satisfies

$$
\mu(\eta)=\infty
$$

for $\eta$ smaller than the threshold for fault tolerance in $d$ dimensional quantum computers, i.e. $\eta<\eta_{0}(d)$.

\section{Other Quantum Systems}

The model of a noisy quantum computer actually holds not only for quantum systems designed to serve as computational devices, but for a much broader class of physical systems as well. We first claim that putting aside the noise process, any $d$ dimensional quantum system, in which the particles are located in space with low enough density, and in which interactions occur only between particles which are not too far apart, can be modeled by a quantum circuit. This can be done by discretizing the medium to very small cells, such that each cell contains at most one particle. Time will be discretized to sufficiently small intervals such that a particle can only move to a neighbor cell in one time step. Then, the movement of particles can be modeled by an interaction between an occupied and an unoccupied cell, and since the density of particles is low, one particle never interacts with more than one other particle at the same time, so the notion of quantum gate is appropriate.

The noise model which is used in this paper is quite general as well, when low density or instantaneous interactions systems are considered. During the time interval in which a particle does not participate in any interaction, stochastic collapses are actually equivalent to a process of local decoherence. Assume that each particle interacts with its own independent thermal 
bath, and the Markovian assumption is applied, so that the environment of each particle is renewed each time step. This corresponds to the process in which the off diagonal terms in the density matrix of one particle decay by some factor between two interactions 21. If the decoherence process operates for time $\Delta t$, the $(i, j)$ element of the density matrix, transforms to

$$
\rho_{i, j} \longmapsto \rho_{i, j} \exp \left(-\gamma \Delta t\left(1-\delta_{i, j}\right)\right)
$$

If we set $\exp (-\gamma \Delta t)=1-\eta$, we get

$$
\rho_{i, j} \longmapsto(1-\eta) \rho_{i, j}+\eta \rho_{i, j} \delta_{i, j},
$$

which is equivalent to a measurement with probability $\eta$. Similarly, the depolarization process can be presented as a gradual change of the density matrix of one particle.

The above arguments show that the model of noisy quantum circuits which we are discussing is interesting as a representative of the class of quantum systems with macroscopically many finite state particles, local instantaneous interactions and local decoherence noise. The analysis done in this paper regarding upper bounds on the entanglement length in the super-critical phase goes through, and therefore theorem 1 and corollary 1 can be generalized to this case.

In such quantum systems, our analysis provides an explanation to the emergence of macroscopic classical behavior above the critical noise rate, as will be discussed in the conclusions.

\section{Experimental Verification}

Unfortunately, we do not yet have a physical realization of a quantum computer of more than several qubits, on which the existence of a phase transition in entanglement length in fault tolerant quantum computer can be verified. However, for the more general case of quantum systems with local interactions and local noise, satisfying the requirements of section 9 , the bound on entanglement length in the super-critical regime can in principle be experimentally testable. What is needed to perform such an experiment is to be able to measure with high enough accuracy the joint density matrix of two subsets of the system. The entanglement between the two sub-systems can then be numerically approximated, using a (doable, but extremely difficult) minimization over equation 2. The entanglement can then be found as a function of the distance between the sets, from which the entanglement length can be deduced. An extremely interesting open problem is to give a concrete design for such an experiment, for an existing quantum system, and to compare the outcomes with the entanglement length predicted by the percolation process.

\section{Quantum-Classical Transition}

The results presented here suggest that the emergence of classical macroscopic phenomena in large quantum systems can be attributed, in certain cases, to the fact that the noise rate is larger than a certain critical point, so that the entanglement length is finite. However, we merely introduced in this paper a new phenomena. The list of questions which remain open is extremely large, and varies on different physical fields.

The first and most basic question should be how general these results are. In this paper, we have been able to show the existence of a non trivial sub-critical phase in fault tolerant quantum computers. Are there more natural quantum systems, in particular systems which are homogeneous (or periodic) in space and in time, which have local interactions and local noise, which are able to maintain long range entanglement in the presence of weak or zero local noise for a long time? Does a random quantum system, i.e. in which random interactions are applied, exhibit long range quantum correlations? Such systems will provide more examples for quantum systems in which a phase transition in entanglement length occurs.

It should be noted here that the notion of zero noise rate does not trivially coincide with that of zero temperature, i.e. long range entanglement in the ground state of the Hamiltonian of the system. An important observation is that the model discussed here deals with non-equilibrium quantum systems. The quantum systems we consider here can be in a steady state, but the density matrix is not in the Gibbs distribution of the eigenvectors of some Hamiltonian. The reason for this is that we did not allow the system to approach equilibrium. Our noise model, or the interaction with the thermal bath, is limited to local interactions. This is a crucial ingredient that causes the phase transition. It is the fact that two forces of even power compete: local interactions in the system against local interactions with the environment, that gives the critical noise rate. The fact that the quantum computer does not achieve equilibrium despite the noise is explained by the fact that the system is cooled constantly by quantum error corrections. It is left as an open problem to further investigate possible equilibrium phase transitions in entanglement, and the connection to the non-equilibrium 
phase transition presented here. The reader is referred to 18 and references therein for an introduction to non-equilibrium phase transitions. We view the fact that we did not allow the system to achieve equilibrium as very important in the derivation here.

Indeed, it is worthwhile to ask which of our assumptions regarding the properties of the physical system are essential, and which are technical. Intuitively, the locality of both noise and interactions seems crucial. This is true since this locality is what makes the two competing forces, the interactions which tend to entangle the system and the noise which tends to disentangle it, comparable in power, which gives rise to the phase transition. It seems, however, that the assumption on the exact form of the noise process might not be so important, and neither is the discretization of the interactions. An important open problem is to relax the assumptions used in this paper, and to generalize the results presented here to other noise models, and to the continuous case. In particular, it is not clear how to generalize the results to the case in which the particle interacts simultaneously with all its neighbors and the environment. It seems that a considerably different approach would be needed in this case.

If the phenomena of the phase transition in entanglement is indeed general, its effect on our understanding the transition from quantum to classical physics needs to be deeply understood. One important question is whether there exists some classical or quasiclassical description of the behavior of a quantum system in its super-critical phase. Another, related, question is whether the existence of a phase transition in entanglement induces other quantum phase transitions at the same critical point in the same system.

A set of open questions regarding the phase transition comes from statistical physics. For example, what are the critical exponents related to this phase transition? What is the universality class of this phase transition? In fact, it is not clear that there is only one critical point here. In the case of the quantum computer, or other quantum systems, there might well be an intermediate regime of noise, in between the two thresholds, for which the entanglement behaves in a different way, i.e. its dependence on the distance is neither an exponential decay nor constant. The question of showing that there is only one critical point at which a transition occurs from exponential decay to independence on the distance remains open.

A very interesting problem is to come up with a better order parameter related to entanglement, rather than the entanglement length. There are many problems with the entanglement length as an order param- eter. The most important one is that it might be that the system is very entangled, but the entanglement between two distant subsets is zero. Such is for example a system in the state $\frac{1}{\sqrt{2}}\left(\left|0^{n}\right\rangle+\left|1^{n}\right\rangle\right)$, for which any subsystem is non-entangled. Entanglement in such very entangled quantum systems will not be detected when looking at sub-systems, and the entanglement length will therefore contain no information about the actual behavior of the entanglement in the system. Another motivation for this question is provided by [2], where the sizes of the clusters is analyzed relaxing the assumptions of nearest neighbor interactions. The sizes of the clusters in this case indeed transform from logarithmic to linear at a critical noise rate. However, the notion of entanglement length cannot be defined in a system without geometry, so it is not clear how to define an order parameter which exhibits the phase transition in this case.

To summarize, we have discovered a phenomena of a phase transition in entanglement in quantum computers, and in general in quantum systems with local decoherence and local interactions which are able to generate long range entanglement in the absence of noise. The suggestion to explain the transition from quantum to classical macroscopic behavior as a phase transition in entanglement is fundamentally different from the standard point of view of gradual transition, usually explained by decoherence. Our results have experimental implications, and raise a long list of open problems related to the foundations of quantum mechanics, as well as to quantum statistical physics.

\section{Acknowledgments}

I am most grateful to Michael Ben-Or and to Michael Nielsen. Discussions with them inspired this work. I am also in debt to Joseph Imri, David Ruelle, and Wojtek Zurek, for interesting discussions. I would like to thank Jennifer Chayes, Christian Borgs, Jeong Han Kim, David Aldous and Oded Schramm for useful comments about classical percolation. Thanks to Julia Kempe, Daniel Lidar and Michael Nielsen for useful comments and corrections on early drafts of this paper.

\section{References}

[1] Aizenman M and Barsky D J, Sharpness of the phase transition in percolation models, Commun. Math. Phys 108 489-526, 1987 
[2] Aharonov D and Ben-Or M, Polynomial Simulations of Decohered Quantum Computers quantph/9611029), 37th Annual Symposium on Foundations of Computer Science (FOCS) pp 46-55, 1996

[3] Aharonov D and Ben-Or M, Fault-Tolerant Quantum Computation with Constant Error, Proc. of the 29th Annual ACM Symposium on Theory of Computing (STOC), Journal version: FaultTolerant Quantum Computation with Constant Error Rate, submitted to SIAM journal of computation.

[4] Bell J S On the Einstein-Podolsky-Rosen paradox, Physics 1 195-200, 1964

[5] Bell J S On the problem of hidden variables in quantum theory, Rev. Mod. Phys. 38 447-52, 1966 Speakable and unspeakable in quantum mechanics 1987 (Cambridge University Press)

[6] Bennett C, Bernstein H, Popescu S, Schumacher B, Concentrating Partial Entanglement by Local Operations, quant-ph/9511030, Phys. Rev. A53, 2046, 1996

[7] Bennett C, DiVincenzo D, Smolin J and Wootters W. Mixed State Entanglement and Quantum Error Correction, Phys.Rev. A54 (1996) 3824-3851

[8] Chayes J T, Puha A and Sweet T, Independent and dependent percolation, IAS/Park City Mathematics Series, Vol. 6, Probability Theory and Applications (E. Hsu and S. Varadhan, eds.), AMS 1999.

[9] David P. DiVincenzo, Tal Mor, Peter W. Shor, John A. Smolin, Barbara M. Terhal, Unextendible Product Bases, Uncompletable Product Bases and Bound Entanglement, quant-ph/9908070

[10] D. Giulini, E. Joos, C. Keifer, J. Kupsch, I.-O. Stamatescu, and H. D. Zeh, Decoherence and the appearance of a classical world in quantum theory, Springer Verlag Berlin Heidelberg 1996.

[11] Einstein A, Rosen N and Podolsky B, Phys. Rev. 47, 7771935

[12] Feller, An introduction to Probability Theory and its Applications, Vol I, Wiley (1961) PP 275-276.

[13] D. Gottesman, "Fault-Tolerant Quantum Computation with Local Gates," to appear in special issue of J. Modern Optics, quant-ph/9903099.

[14] G. Grimmett, Percolation, Springer-Verlag, New York 1989.
[15] Michal Horodecki, Pawel Horodecki, Ryszard Horodecki, Mixed-state entanglement and distillation: is there a "bound" entanglement in nature? Phys.Rev.Lett. 80 (1998) 5239-5242

[16] K Kitaev A Yu, Quantum Computations: Algorithms and Error Corrections, in Russian Math. Surveys, 52:6, 1191-1249

[17] Knill E, Laflamme R, and Zurek W, Resilient quantum computation, Science, vol 279, p.342, 1998, see also: Knill E, Laflamme R and Zurek W H 1997 Resilient quantum computation: error models and thresholds, in LANL e-print quantph/9702058, http://xxx.lanl.gov (1997), in Proceedings of the royal society of London $A$. and Knill E and Laflamme R, Concatenated quantum codes, in LANL e-print quant-ph/9608012, http://xxx.lanl.gov (1996)

[18] J. Marro and R. Dickman, Nonequilibrium Phase Transitions in Lattice Models, Cambridge University Press, 1999.

[19] Menshikov M V, Molchanov S A and Sidorenko A $\mathrm{F}$, Percolation theory and some applications, Itogi Nauki $i$ Techniki (Series of Probability Theory, Mathematical Statistics, Theoretical Cybernetics) 24 53-110, 1986

[20] Nielsen M. A, Continuity Bounds for Entanglement, quant-ph/9908086

[21] Palma G M, Suominen K-A \& Ekert A K 1996 Quantum computers and dissipation, Proc. Roy. Soc. Lond. A 452 567-584

[22] Rains E, A rigorous treatment of distillable entanglement, quant-ph/9809078.

[23] Shor P W, Polynomial-time algorithms for prime factorization and discrete logarithms on a quantum computer, SIAM J. Comp., 26, No. 5, pp 1484-1509, October 1997

[24] Zurek W H, Decoherence and the transition from quantum to classical Physics Today 44(10), October, 1991 36-44. 